\title{
A syphilis epidemic among Black MSM in the context of endemic HIV: what do the venue affiliation networks look like over time?
}

\author{
Carla Tilchin ${ }^{1}$ Christina Schumacher ${ }^{1,2}$ Carl Latkin $^{3}$ Khalil Ghanem ${ }^{4}$ LaNisha Childs ${ }^{2}$ \\ Matthew Hamill ${ }^{4}$ Suzanne Grieb ${ }^{1}$ Kathleen Page ${ }^{2,4}$ Adena Greenbaum$^{2}$ Jacky M Jennings ${ }^{1,3}$ \\ ${ }^{1}$ Center for Child and Community Health Research (CCHR), Department of Pediatrics, Johns Hopkins School of Medicine \\ 2 Baltimore City Health Department \\ ${ }^{3}$ Department of Health, Behavior, and Society, Johns Hopkins Bloomberg School of Public Health \\ ${ }^{4}$ Department of Medicine, Johns Hopkins School of Medicine
}

\section{Background}

- Baltimore, Maryland has one of the most severe syphilis epidemics with a cooccurring HIV epidemic among Black men who have sex with men (MSM).

- Key to transmission dynamics is the presence of disease and network structures to support infection propagation.

- Sex partner meeting places can be used as points of access for interventions and the network structure of meeting places may have important implications for epidemics.

- The objective was to describe temporal changes in the network structure of venue affiliation networks of syphilis and/or HIV infected MSM over five years during a local MSM syphilis epidemic.

\section{Methods}

- Public health surveillance data from MSM diagnosed with syphilis and/or HIV and reporting $\geq 1$ sex partner meeting venue from 2012-2016 were examined.

- Venue affiliation network graphs and whole network structure measures (density, compactness) were generated using UCINET to assess whole network structure changes over time.

- Density: a measure of the total number of ties divided by the total number of possible ties

- Compactness: an average reciprocal measure of the distances and geodesic distances (shortest paths) between nodes. Compactness is 1 when all nodes are adjacent to each other.

\section{Results}

- 497 syphilis and/or HIV infected MSM were included (syphilis only $30.1 \%$ $(n=154)$, HIV only $27.4 \%(n=136)$, both $42.5 \%(n=207)) .63 .8 \%(n=317)$ were Black MSM. 137 unique venues were nominated, of which 55 venues were reported by $>1 \mathrm{MSM}$ and were included in this analysis.

- Comparing 2012 to 2016 , the density of the network increased $23.8 \%$ (0.09 to 0.11$)$, and the compactness of the network increased $16.3 \%(0.22$ to 0.26$)$ (Figure 1)

- The proportion of venues nominated by MSM with HIV and/or syphilis (vs. only one diagnosis) in the main network component increased $17.1 \%$ (80.0\% to $93.7 \%$ ) (Figure 2 )

\section{Conclusions}

- Venue affiliation graphs of sex partner meeting places of syphilis and/or HIV infected MSM suggest a discrete set of potential intervention places and increasing co-nomination, density and compactness of the venue network structure during syndemics of syphilis and HIV.

- The network structure changes over time may suggest higher rates of transmission and increased difficulty of interrupting network transmission through fragmentation.

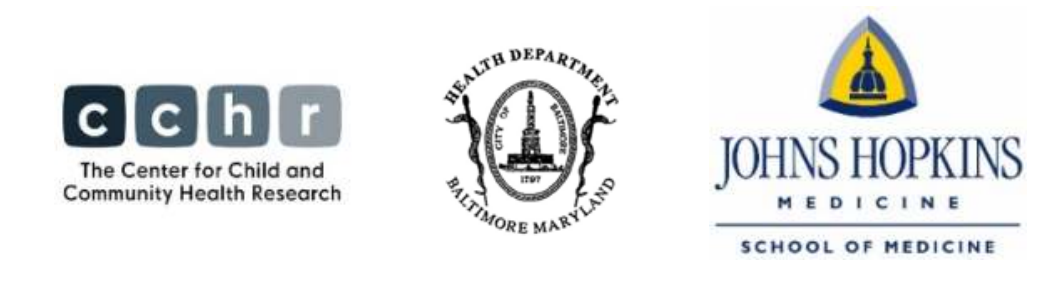

Figure 1. Measures of density and compactness of the venue affiliation networks of MSM diagnosed with syphilis and/or HIV, Baltimore City, 2012-2016.

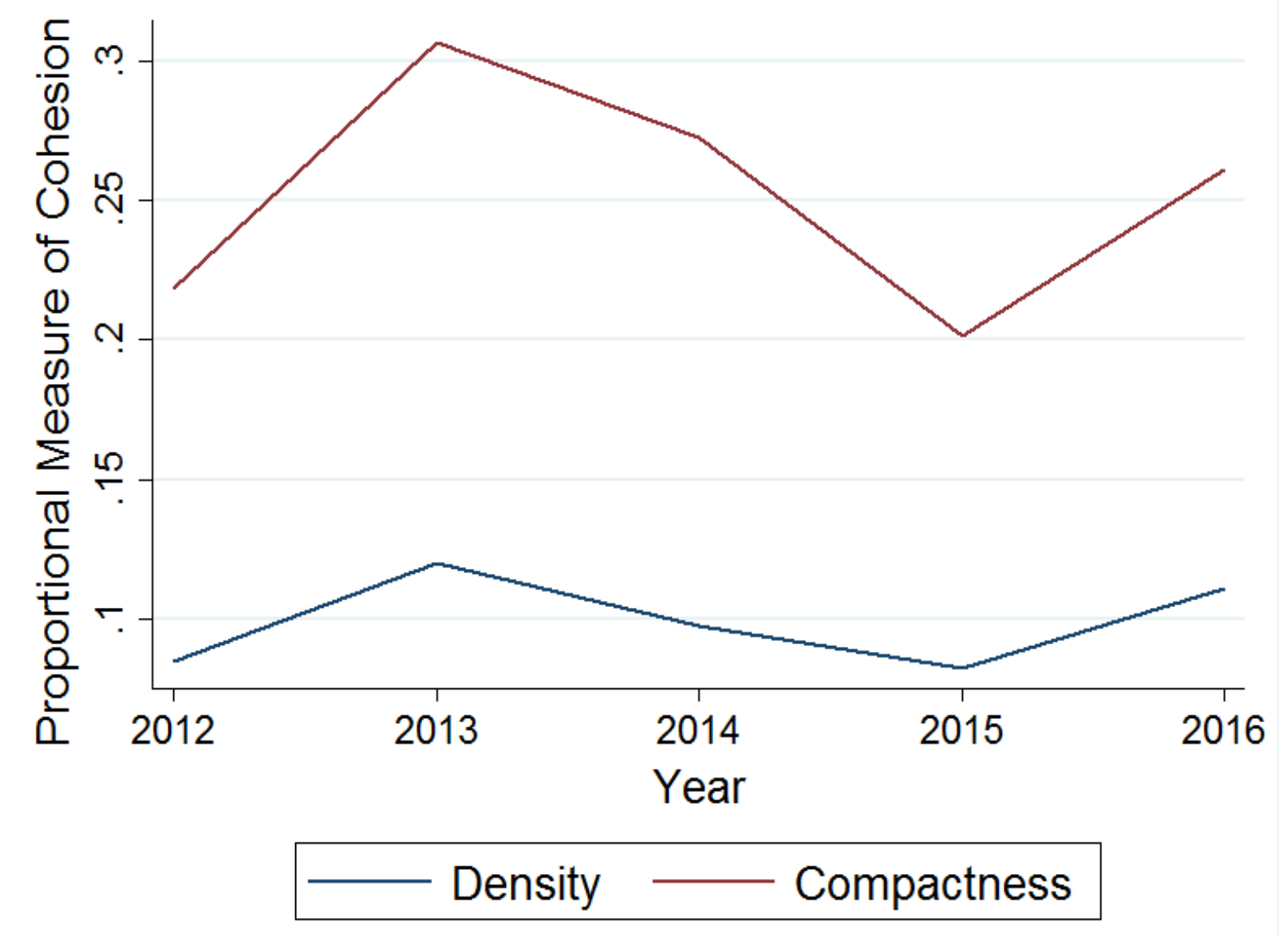

\begin{tabular}{lll} 
Year & Density & Compactness \\
\hline 2012 & 0.08 & 0.22 \\
2013 & 0.12 & 0.31 \\
2014 & 0.10 & 0.27 \\
2015 & 0.08 & 0.20 \\
2016 & 0.11 & 0.26 \\
\hline
\end{tabular}

Figure 2. The main component of the venue affiliation network graph among MSM diagnosed with syphilis and/or HIV, Baltimore City, 2012 (2A) and 2016 (2B). Squares represent venues.

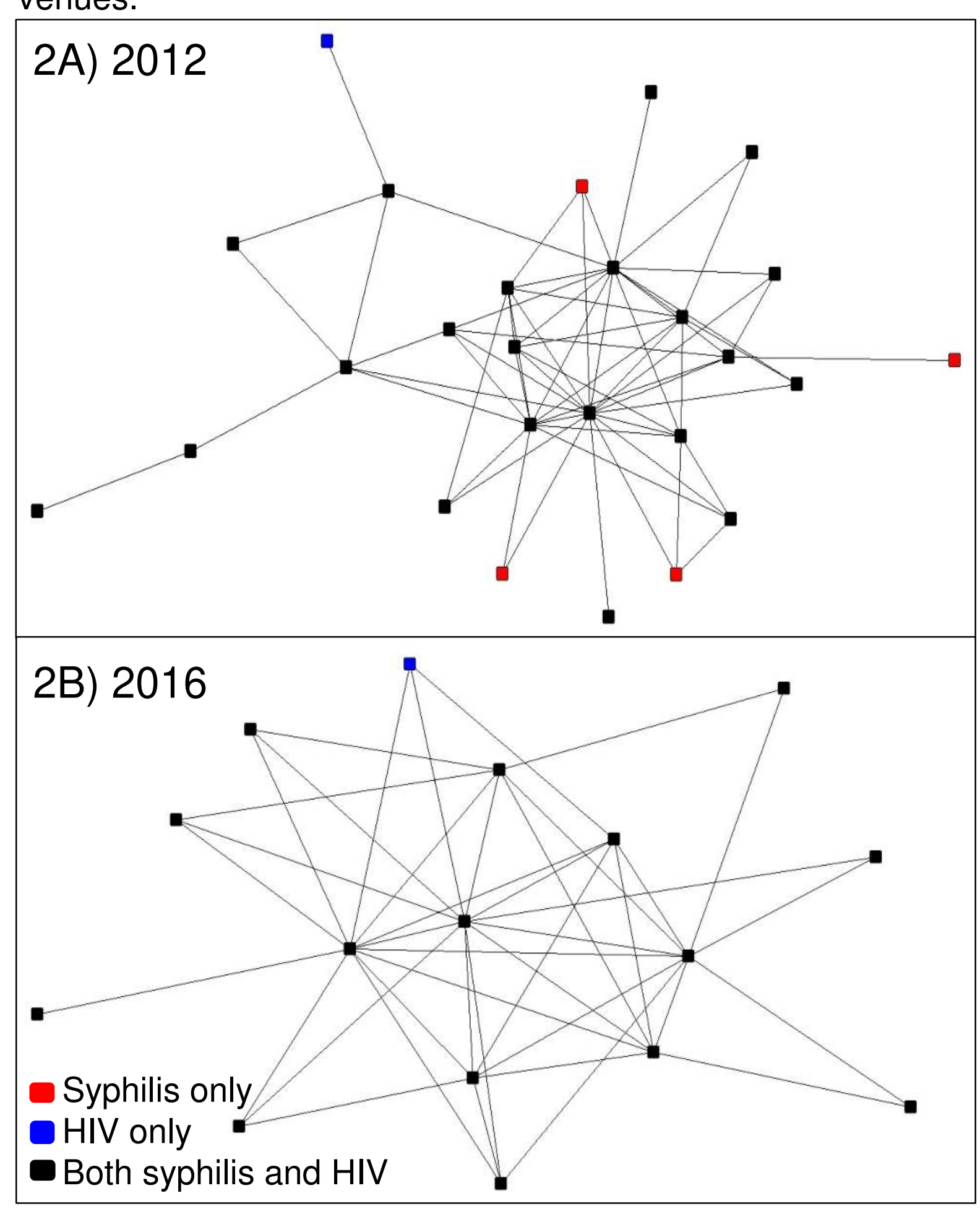

